\title{
Camera Self-calibration with Parallel Screw Axis Motion by Intersecting Imaged Horopters
}

\author{
Ferran Espuny ${ }^{1}$, Joan Aranda ${ }^{2}$, and José I. Burgos Gil ${ }^{3}$ \\ 1 Dépt. Images et Signal, GIPSA-Lab, Grenoble-INP \\ Ferran.Espuny@gipsa-lab.grenoble-inp.fr \\ 2 Dept. of Automatic Control and Computing Engineering, UPC \\ Joan. Aranda@upc . edu \\ 3 Instituto de Ciencias Matemáticas, CSIC-UAM-UCM-UC3 \\ jiburgosgil@gmail.com
}

\begin{abstract}
We present a closed-form method for the self-calibration of a camera (intrinsic and extrinsic parameters) from at least three images acquired with parallel screw axis motion, i.e. the camera rotates about parallel axes while performing general translations. The considered camera motion is more general than pure rotation and planar motion, which are not always easy to produce. The proposed solution is nearly as simple as the existing for those motions, and it has been evaluated by using both synthetic and real data from acquired images 1
\end{abstract}

\section{Introduction}

We say that a camera follows a parallel screw axis motion when it rotates about axes parallel to a fixed screw direction, while translating in any direction neither orthogonal nor parallel to that direction. We consider in this paper the self-calibration problem from three or more images acquired by a camera with unchanging internal parameters undergoing a parallel screw axis motion.

By self-calibration, we mean the calibration of a camera without any knowledge on the scene or the camera pose (location and orientation) [116].

A scene can be reconstructed up to a projective ambiguity (projective reconstruction) by using two views of the scene. This can be done by using the fundamental matrices between pairs of views, or, alternatively, multiple view tensors. We will follow a stratified approach for self-calibration, consisting in looking first for an affine reconstruction, and then upgrading it to a Euclidean reconstruction. We can achieve the affine level by determining the relative infinite homographies, and the Euclidean one through the calibration of the camera.

The most simple methods for the Euclidean self-calibration of a camera correspond to particular camera motions: pure rotations [9] and planar motion 117 1215. However, since the camera centre is not visible, the assumption of pure rotation of a camera is only plausible for distant scenes. The assumption of planar motion requires in turn the relative translations of the camera to be orthogonal to a fixed screw direction. We relax the previous motion constraints,

${ }^{1}$ Research supported by the Spanish MICINN project MTM2009-14163-C02-01.

A. Heyden and F. Kahl (Eds.): SCIA 2011, LNCS 6688, pp. 1-12, 2011.

(C) Springer-Verlag Berlin Heidelberg 2011 
by allowing the camera to translate in more general directions (for three views, one relative planar motion will yet be allowed).

The parallel screw axis camera motion is known to be degenerate for direct self-calibration methods [17]: there exists a uni-parametric family of possible conics consistent with the displacements of the camera. The existing general stratified self-calibration methods could be used with this camera motion, but some require the performance of a 3-dimensional search [1013 15, and the closedform ones [14168] do not guarantee the uniqueness of the solution, having all of them a high degree of complexity. In contrast, we give in this paper a simple closed-form (unique) solution to the stratified self-calibration problem with parallel screw axis camera motion.

The horopter curve of two images is the set of space points projecting onto two points of identical coordinates in both images. Under general motion, the horopter is a twisted cubic that projects on a non-degenerate conic, given by the symmetric part of the fundamental matrix. Horopter curves have already been used for camera self-calibration with general [15] and planar 17/5] motions.

The intersection of the imaged horopters corresponding to three views will allow us to determine: the apex (image of the screw axis direction) and the image of the two circular points, which give the metric on the planes orthogonal to the screw axes. Similarly to planar motion, the scene can be recovered up to a 1-D affinity in the screw direction. To resolve this ambiguity, some assumptions on the camera pixel geometry can be made that lead us to a complete camera calibration (zero-skew constraint, known image aspect ratio or known principal point); the screw direction conditions the necessary additional assumptions [20]2].

In Section 2 we state the basis for understanding our method and give a characterisation of the apex, which we will use for the self-calibration in some particular cases. In Section 3 we formulate the problem and deduce the constraints used for its resolution. We detail our stratified solution in Section 4. the affine step consists of a closed-form solution with an optional iterative refinement, and the linear Euclidean upgrade uses the knowledge of the apex and imaged circular points. Finally, experimental results and conclusions are given.

Notation. We will use $\simeq$ to denote an equality up to scale factor, for the matrix-vector and matrix-matrix products, $\times$ for the cross product of 3 -vectors, and $[u]_{\times}$for the matrix associated to a 3-vector $u$ so that $[u]_{\times} \cdot v=u \times v, \forall v$.

\section{Background}

The general contents of this section can be found in [6]11; extra references are given in the text. Let us consider a set of two images of a rigid scene acquired from different locations and with different orientations by a camera with intrinsic parameters given by a calibration matrix

$$
K=\left(\begin{array}{rrr}
\alpha_{u} & s & u_{0} \\
& \alpha_{v} & v_{0} \\
& & 1
\end{array}\right)=\left(\begin{array}{rr}
f k_{u}-f k_{u} \cot \varphi & u_{0} \\
f k_{v} / \sin \varphi & v_{0} \\
& 1
\end{array}\right)
$$


being $f$ the focal length, $\left(u_{0}, v_{0}\right)$ the image principal point, $k_{v} / k_{u}$ the aspect ratio, $s$ the skew parameter, and $\varphi$ the angle between the camera sensor axes.

The affine knowledge of the scene, codified by the plane at infinity $\pi_{\infty}$, allows us to recognise parallel lines and to compute affine ratios of aligned points. The Euclidean knowledge of the scene, codified by the absolute conic $\Omega_{\infty}$, allows us to compute distances up to scale and angles. We denote by $\left(\begin{array}{cc}R & t \\ 0^{T} & 1\end{array}\right)$ the relative displacement, taking the second camera to the first one. We assume that the relative angle of rotation $\theta$ satisfies $0<\theta<\pi$ and that the relative translation satisfies $t \neq 0$ (we exclude pure translations and pure rotations). We denote by $I, J, r$ the three fixed points of the relative displacement (eigenvalues of $R$ ), which span the plane at infinity. The circular points $I, J \in \Omega_{\infty}$ are orthogonal to $r$, and codify the metric of the planes orthogonal to $r$. We denote by $\tilde{I}, \tilde{J}, v$ the images of those three points: $\tilde{I} \simeq K \cdot I, \tilde{J} \simeq K \cdot J, v \simeq K \cdot r$.

\subsection{Stratified Self-calibration}

A reconstruction of the scene and cameras is called projective, affine or Euclidean when it corresponds with the real scene and cameras via a projectivity, affinity or similarity, respectively.

The fundamental matrix $F$ represents the epipolar map assigning to a point on the first image the epipolar line of possible corresponding points on the second image; $F^{T}$ represents the epipolar map in the inverse image order. These maps are not defined in the epipoles $e, e^{\prime}$, right an left null-spaces of $F$, respectively. The fundamental matrix is determined in general by seven or more correspondences. Its knowledge is equivalent to knowing a projective reconstruction.

The infinite homography $H_{\infty}$ is the homography between the two images induced by the plane at infinity $\pi_{\infty}$. The matrix $F$ can be decomposed as:

$$
F \simeq\left[e^{\prime}\right]_{\times} \cdot H_{\infty}
$$

The knowledge of $H_{\infty}$ allows us to upgrade a projective reconstruction to an affine one. In fact, the fixed points of the infinite homography are the apex $v$ and the imaged circular points $\tilde{I}, \tilde{J}$, which are image of three points on the plane at infinity. By construction, we have that $H_{\infty} \simeq K \cdot R \cdot K^{-1}$ or, equivalently:

$$
H_{\infty} \simeq(\tilde{I} \tilde{J} v) \cdot \operatorname{diag}(\exp (-i \theta), \exp (i \theta), 1) \cdot(\tilde{I} \tilde{J} v)^{-1} .
$$

The image of the absolute conic, denoted by $\omega_{\infty}$, is a conic on the image plane codifying, up to scale, the metric on this plane. We denote by $\omega_{\infty}^{*}$ its dual. Both conics are equivalent to the camera calibration, since $\omega_{\infty} \simeq\left(K \cdot K^{T}\right)^{-1}$, $\omega_{\infty}^{*} \simeq K \cdot K^{T}$, and allow us to upgrade a projective reconstruction to a Euclidean one. It follows from $H_{\infty} \simeq K \cdot R \cdot K^{-1}$ and $\omega_{\infty} \simeq\left(K \cdot K^{T}\right)^{-1}$ that $\omega_{\infty}$ is invariant under the infinite homography $H_{\infty}$. In fact, using (3), we obtain that, for some $\lambda \neq 0$, it holds [20]:

$$
\omega_{\infty}^{*} \simeq\left(\tilde{I} \cdot \tilde{J}^{T}+\tilde{J} \cdot \tilde{I}^{T}\right)+\lambda v \cdot v^{T}
$$


In conclusion, we can follow a stratified approach to camera calibration from the fundamental matrix $F$ : first, we determine the infinite homography $H_{\infty}$ (affine self-calibration); then, we linearly determine $\omega_{\infty}$, the image of the absolute conic, which is invariant under that homography (Euclidean self-calibration).

\subsection{Horopter Curves}

The horopter curve of two images is the set of space points that project onto points of identical (i.e. proportional) coordinates in both images. The horopter curve passes through the camera centres and through the fixed points of the relative displacement, which include $r$, the point at infinity of the rotation axis, and the circular points $I, J$. For a general camera motion, it is a proper (irreducible) twisted curve. It decomposes as three lines when $t$ is parallel to $r$, and as a circle plus a line (screw axis), when $t$ is orthogonal to $r$ (planar motion).

The image of the horopter is a conic, non-degenerate a for general camera motion and degenerate (two lines) otherwise. Since the image of the horopter is the locus of corresponding points with identical coordinates, it is a conic with matrix given by the symmetric part of the fundamental matrix:

$$
F_{\text {sym }}:=\frac{1}{2}\left(F+F^{T}\right) .
$$

This conic will contain the apex $v$ and $\tilde{I}, \tilde{J}$, the image of the circular points.

The ratios of intersection of the horopter curves with the plane at infinity have been exploited for the self-calibration with general camera motion in [15. We will use a similar constraint for an auxiliary characterisation of the apex:

Lemma 1. If $F_{\text {sym }}$ is non degenerate and $\mu$ is a scalar such that

$$
F \cdot \tilde{I}=\mu \exp (-i \theta) e^{\prime} \times \tilde{I}
$$

then the apex is the only point $v \in F_{\text {sym }}$ satisfying

$$
F \cdot v=\mu e^{\prime} \times v .
$$

Proof. Both equations follow from (3) and (2). If we had $v^{\prime} \neq v$ satisfying (7) then, for every $\lambda \in \mathbb{R}$ we would have $F \cdot\left(v+\lambda v^{\prime}\right)=\mu e^{\prime} \times\left(v+\lambda v^{\prime}\right)$, and thus $\left(v+\lambda v^{\prime}\right)^{T} \cdot F \cdot\left(v+\lambda v^{\prime}\right)=0$. Hence, the conic $F_{\text {sym }}$ would contain the line $v \times v^{\prime}$ and it would be degenerate.

\section{Problem Statement and Constraints}

Remember that we say that a camera follows a parallel screw axis motion when it rotates about axes parallel to a screw direction while translating in any direction neither orthogonal nor parallel to that direction. Assume that we know three fundamental matrices $F^{i, j}, 1 \leq i<j \leq 3$ corresponding to three images 
acquired from different orientations by a camera with unchanging internal parameters undergoing a parallel screw axis motion. We are interested in solving the stratified self-calibration of the camera, as explained in Section 2 .

Accordingly, we want to determine three points in the image: the apex $v$ and the imaged circular points $\tilde{I}, \tilde{J}$, which are common to the three views:

Theorem 1 (Intersection of the Imaged Horopters). Under parallel screw axis camera motion, the three different imaged horopters (conics represented by the symmetric parts of the fundamental matrices) intersect at the images $\tilde{I}, \tilde{J}$ of the circular points (which are complex conjugate points) and the (real) apex $v$. A fourth real intersection point can exist under particular camera motions.

Proof. The screw direction $r$ of the rotation axes and the circular points $I, J$ are common to all the camera relative displacements. Therefore, their images belong to the imaged horopters. Five or more intersection points can not exist for the three imaged horopters, since then these conics would be coincident, and the relative displacements would be all the same. In particular, the intersection can not contain a fourth non-real intersection point (and its conjugate).

Observe that the three fundamental matrices $F^{i, j}$ corresponding to three views of a common scene satisfy three compatibility constraints [6]11]

$$
\left(e^{2,3}\right)^{T} \cdot F^{1,2} \cdot e^{1,3}=\left(e^{3,2}\right)^{T} \cdot F^{1,3} \cdot e^{1,2}=\left(e^{3,1}\right)^{T} \cdot F^{2,3} \cdot e^{2,1}=0 .
$$

Using the infinite homographies $H_{\infty}^{i, j}$ to parametrise the fundamental matrices, these constraints reduce to a single one:

Theorem 2 (Three-View Affine Compatibility). Consider the infinite homographies $H_{\infty}^{i, j}$, epipoles $e^{i, j}$ and fundamental matrices $F^{i, j}$ such that

$$
\begin{aligned}
F^{i, j} & \simeq\left[e^{j, i}\right]_{\times} \cdot H_{\infty}^{i, j} \\
e^{j, i} & \simeq H_{\infty}^{i, j} \cdot e^{i, j} \\
H_{\infty}^{2,3} & \simeq H_{\infty}^{1,3} \cdot\left(H_{\infty}^{1,2}\right)^{-1} .
\end{aligned}
$$

The three compatibility equations (8) are satisfied if, and only if, any of the following set of points is linearly dependent:

$$
\begin{aligned}
& \left\{e^{1,2}, e^{1,3},\left(H_{\infty}^{1,2}\right)^{-1} \cdot e^{2,3}\right\} \subset \mathcal{R}_{1}, \\
& \left\{e^{2,1}, e^{2,3}, H_{\infty}^{1,2} \cdot e^{1,3}\right\} \subset \mathcal{R}_{2}, \\
& \left\{e^{3,1}, e^{3,2}, H_{\infty}^{2,3} \cdot e^{2,1}\right\} \subset \mathcal{R}_{3},
\end{aligned}
$$

where $\mathcal{R}_{i}$ denotes the $i$-th image plane.

Proof. By (10) and (11), the sets (13) and (14) are the result of applying $H_{\infty}^{1,2}$ and $H_{\infty}^{1,3}$, respectively, to the set (12). Hence, the linear dependence of any of those sets is equivalent to the linear dependence of all of them. The direct 
substitution of the parametrisation (9) into (8) gives directly two of the linear dependency conditions; for instance, the first term in (8) is

$$
\left(e^{2,3}\right)^{T} \cdot F^{1,2} \cdot e^{1,3} \stackrel{\sqrt[9]{\simeq}}{\simeq}\left(e^{2,3}\right)^{T} \cdot\left[e^{2,1}\right]_{\times} \cdot H_{\infty}^{1,2} \cdot e^{1,3},
$$

which is zero if, and only if, the vectors in (13) are linearly dependent.

\section{Self-Calibration Method}

We will solve the affine self-calibration problem by intersecting the imaged horopters (Theorem1), given by the symmetric part of the fundamental matrices $F^{i, j}, 1 \leq i<j \leq 3$. We propose a two-step method for computing this intersection: a closed-form solution is obtained and further refined using Theorem 2 We also revisit the upgrading step from affine to Euclidean self-calibration.

\subsection{Affine Self-calibration by Intersecting the Imaged Horopters}

Given the three fundamental matrices $F^{i, j}, 1 \leq i<j \leq 3$, we can compute the imaged horopters. To intersect them, we will follow a least squares approach, which we obtain by adapting the method in the Appendix of [3] to our constraints: the conics intersect in three points, one real and two complex conjugate.

General Intersection. Assume that we have a pair of conics $C, C^{\prime}$ with coefficients in $\mathbb{R}$, intersecting in $\tilde{I}, \tilde{J}$ (complex-conjugate pair) and $v, P$ (two points with coordinates in $\mathbb{R}$ ). Each of the three degenerate conics (zero determinant) in the family $C+\mu C^{\prime}$ is a pair of lines joining two points in the intersection of $C$ with $C^{\prime}$. The only lines with real coefficients will be $\tilde{I} \times \tilde{J}$ and $v \times P$. Therefore, if we tale $\mu \in \mathbb{R}$ such that $D=C+\mu C^{\prime}$ has $\operatorname{det} D=0$, then $D$ consists of the line joining the imaged circular points plus a line passing through the apex.

Using the three imaged horopters, we can compute three degenerate conics $D^{k}$ following the described procedure. The apex $v$ will be the unique point in the intersection of the $D^{k}$, and the imaged circular points can be obtained by averaging the intersection of the common line of the three $D^{k}$ with each of the conics (see Figure 1). Note that both computations can be integrated in a leastsquares estimation process in presence of noise, and/or if more than three images are available. Once we have an estimation for the apex $v$ and the imaged circular points $\tilde{I}, \tilde{J}$, the relative rotation angles $\theta^{i, j}$ can be computed using (6).

The previous estimations can be optionally refined by minimising the sum of squares of distances of the image points to the epipolar lines obtained using the fundamental matrices parametrised as follows. By (9), we can use 13 parameters to describe the three fundamental matrices: we can take one coordinate of $\tilde{I}$ to be one ( 4 dof for $\tilde{I}, \tilde{J}), 2$ parameters for the relative rotation angles (since $\left.\theta^{2,3}=\theta^{1,3}-\theta^{1,2}\right)$, unitary vectors for the apex $v$ and the epipoles $e^{j, 1}$ (6 dof), and 1 parameter for $e^{3,2}$ satisfying (14). 


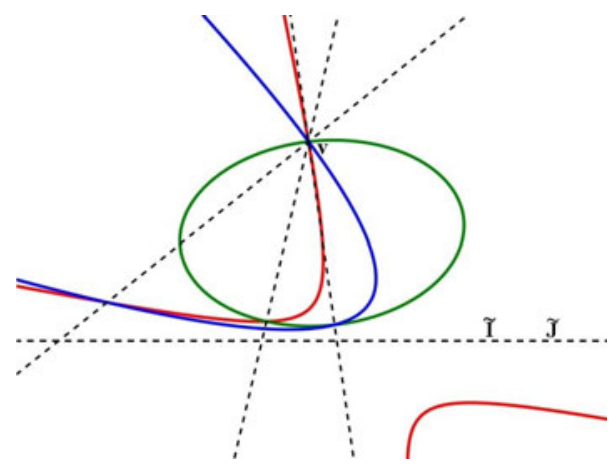

Fig. 1. Imaged horopters corresponding to a general parallel screw axis motion. The discontinuous lines represent the real degenerate conics from each pair-wise linear family of conics. The apex $v$ can be obtained by intersecting the lines not containing $\tilde{I}, \tilde{J}$.

Particular Cases. We excluded in our assumptions the cases of pure translation and planar motion. However, it could happen that the relative displacements are close to these degenerate motions. In the first case, the fundamental matrix is practically anti-symmetrical, and the computation of the imaged horopter is badly conditioned. In the second case, the imaged horopter is degenerate. This is not a problem if only happens for one relative displacement (see Fig. 22 left); otherwise, a planar motion self-calibration method should be used.
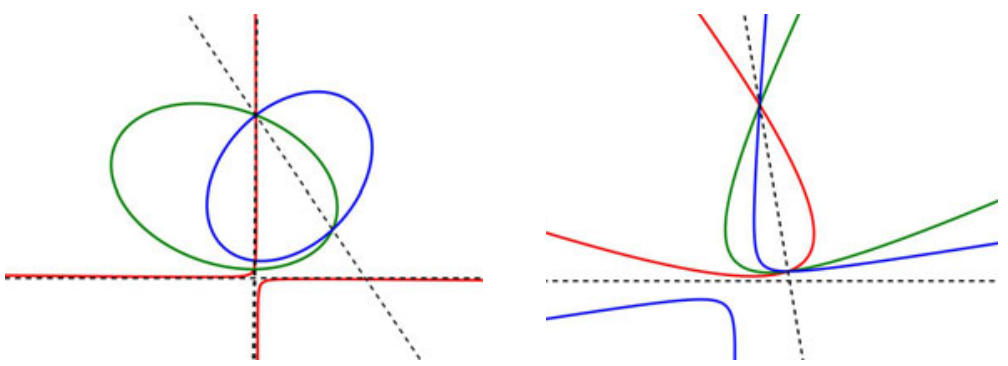

Fig. 2. Left: if one relative displacement is (close to being) planar, the image of the horopter is degenerate (coordinate hyperbola in the picture). Right: an example of three conics intersecting in two real and two complex conjugate points.

As we said in Theorem 1, the three imaged horopters can intersect in four points for particular camera motions. The characterisation of these motions is out of the scope of this paper. In this case, the pair-wise real degenerate conic will be the same for any pair of imaged horopters (see Fig. 2, right). The four intersection points can be obtained by averaging the intersection of such degenerate conic with each imaged horopter. The imaged circular points will be the non-real points, while the apex will be the real point satisfying Lemma 1. 


\subsection{Linear Upgrade from Affine to Euclidean}

Once we know the affine self-calibration, the self-calibration equations are not enough to determine the Euclidean self-calibration under parallel screw axis camera motion: all the conics in the one-dimensional family (4) satisfy those equations. We revisit the additional assumptions usually taken on $\omega_{\infty}^{*}$, showing that they can be imposed linearly.

Observe that by Section 2 we have:

$$
\omega_{\infty}^{*} \simeq K \cdot K^{T}=\left(\begin{array}{ccc}
\alpha_{u}^{2}+s^{2}+u_{0}^{2} & s \alpha_{v}+u_{0} v_{0} & u_{0} \\
s \alpha_{v}+u_{0} v_{0} & \alpha_{v}^{2}+v_{0}^{2} & v_{0} \\
u_{0} & v_{0} & 1
\end{array}\right),
$$

being the coefficients of $K$ denoted as in (11). If the rotation direction $r$ has non zero $x$ and $y$ components, the zero skew assumption $(s=0)$ determines uniquely the conic $\omega_{\infty}^{*}$ as that $\omega^{*}$ in (4) satisfying

$$
\omega_{1,2}^{*} \omega_{3,3}^{*}-\omega_{1,3}^{*} \omega_{3,2}^{*}=0,
$$

where $\omega_{i, j}^{*}=\omega_{j, i}^{*}$ denotes the element in the $i$-th row and $j$-th column of a matrix representing $\omega^{*}$.

However, if the rotation direction $r$ has zero $x$ or $y$ components, then the zero skew constraint (16) is satisfied by every conic in the family (4) [20]2 . In this case, if the rotations are not about axes parallel to the $z$ axis, assuming zero skew, $s=0$, and unit aspect ratio, $\alpha_{u}=\alpha_{v}$, determines uniquely $\omega_{\infty}^{*}$. The equation to impose is the square pixel constraint:

$$
\omega_{1,1}^{*} \omega_{3,3}^{*}-\left(\omega_{1,3}^{*}\right)^{2}=\omega_{2,2}^{*} \omega_{3,3}^{*}-\left(\omega_{2,3}^{*}\right)^{2} .
$$

Apparently, the last two constraints (16) and (17), when imposed on the family (4), give equations quadratic in $\lambda$. We observe though that, since $\left(w \cdot w^{T}\right)_{i, j}=$ $w_{i} w_{j}$, the coefficient of $\lambda^{2}$ in both sides of any of the equations is zero, and consequently those equations are linear in $\lambda$. In fact, by (1) the zero skew constraint imposed on $\omega=\omega_{\infty}$ reads $\omega_{1,2}=0$, and the additional unit aspect ratio constraint reads $\omega_{1,1}=\omega_{2,2}$. Both equations are obviously linear on the coefficients of $\omega_{\infty}$ and therefore equivalent to those for $\omega_{\infty}^{*}$. An optional bundle adjustment step [4] could be used to refine the Euclidean self-calibration.

\section{Experiments}

We conducted experiments with both synthetic and real data in order to evaluate the performance of the self-calibration method of Section 4. We used simulated data to study the behaviour of the method with respect to the pitch and roll angles of the camera for different levels of noise in the initial image correspondences used for the computation of the fundamental matrices. Finally, we applied the method to a set of real images, comparing our results with a ground truth given by the calibration from pattern method in 1819 . 
Synthetic Data In our simulations, we generated a cloud of 100 points uniformly distributed inside a 3 -D sphere. We placed in the space three random cameras related by a parallel screw axis motion in such a way that each camera could view at least half of the sphere. The calibration matrix of the cameras was

$$
K=\left(\begin{array}{ccc}
1000 & 0 & 320 \\
0 & 1000 & 240 \\
0 & 0 & 1
\end{array}\right)
$$

In the Euclidean frame associated to the first camera, the screw direction had coordinates

$$
r=(\sin \alpha \sin \beta,-\cos \alpha \sin \beta, \cos \beta),
$$

where $\beta$ was the pitch angle, considered as the angle between the principal axis of the camera (the $z$ axis) and the screw direction, and $\alpha$ was the roll angle, understood as the angle of rotation of the camera about the $z$ axis. The relative rotation angles, relating any pair of cameras, varied randomly between 10 and 120 degrees. We added Gaussian noise (with deviation $\sigma$ ranging from 0 to 3 pixels) to the projection of the 3 -D points and used the noisy image points as initial data for the self-calibration method. For each combination of values $\alpha$, $\beta$ and $\sigma$ we simulated 1000 times different 3-D points, camera poses and image points; we applied the proposed self-calibration method to each simulation and computed the mean of the obtained errors.

In a first experiment (Figure 3), we fixed the roll angle $\alpha$ to be zero and varied the pitch angle $\beta$. Since $r$ had no $x$ component, we used the unit aspect ratio constraint to achieve a Euclidean reconstruction (Section 4). We observed that
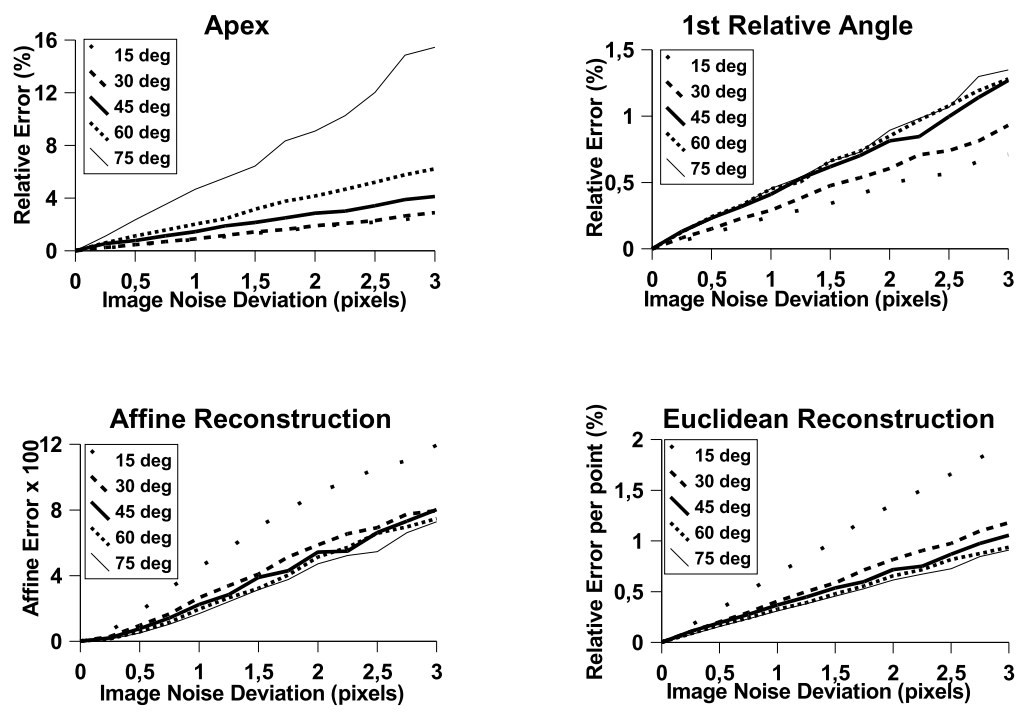

Fig. 3. Errors for roll angle $\alpha=0$ and different values of $\beta$ (degs) and $\sigma$ (pixels) 
the closer the cameras were to being parallel to the screw direction ( $\beta$ small), the more exact was the apex and relative angles computation but the more inexact was the estimation of the image of the circular points and, consequently, the worst were the obtained affine and Euclidean reconstructions.

In a second experiment (Figure 4), we fixed $\beta=60$ degrees and varied the roll angle $\alpha$ from 0 to 45 degrees. According to Section 4 the zero skew constraint was sufficient for the values of $\alpha \neq 0$ in order to upgrade the affine reconstruction to a Euclidean one. We observed that the bigger the angle $\alpha$ was, the better were the estimations of the apex and image of the circular points and, consequently, the better were the obtained affine and Euclidean reconstructions.
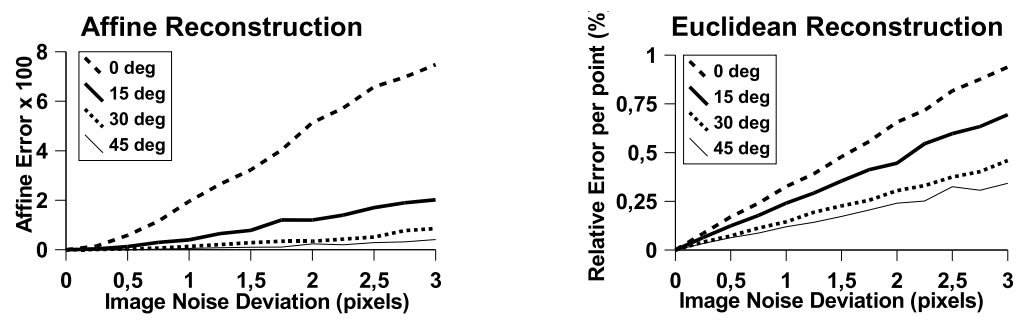

Fig. 4. Errors for $\beta=60$ degs and different values of $\alpha$ (degs) and $\sigma$ (pixels)

Real Images. The images shown in Figure 5 are part of a 5-image sequence (768 $\times 576$ pixels) acquired by a camera mounted on an articulated robotic arm. The screw direction (rotation axis) had no $x$ component and the angle between this direction and the $z$ axis of the camera was approximately equal to 60 degrees.
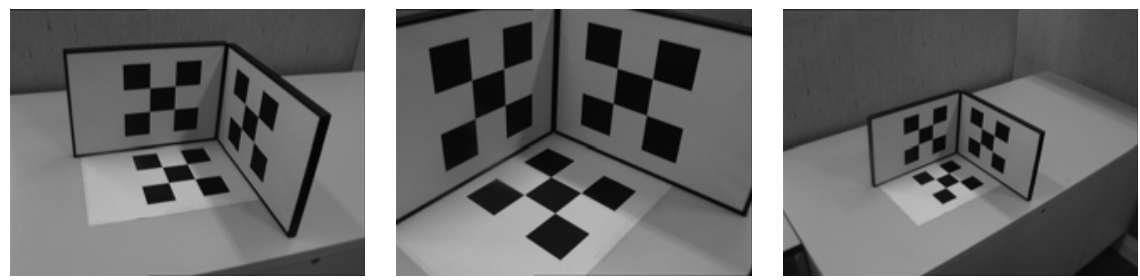

Fig. 5. Image sequence of a single pattern with $\beta=60$ degs, $\alpha=0$ degs (aprox.)

We selected manually the 48 "chessboard" corners from each image and used standard methods to refine their coordinates with sub-pixel accuracy. We first computed the affine self-calibration by intersecting the imaged horopters. Since the $x$ component of the screw direction was zero, we imposed the square pixel constraint (zero skew and unit aspect ratio) to obtain the camera calibration matrix. We describe the results using the notations stated in (1): 
Table 1. Results with the 5 real images, being $\alpha=\alpha_{u}=\alpha_{v}$. First: least-square solution using the 10 imaged horopters. Next: mean, median and deviation of the closed-form solution applied to each of the 10 triplets of images.

\begin{tabular}{c|c|ccc} 
& value & mean & median & deviation \\
\hline$\alpha$ & 863.07 & 864.01 & 862.36 & 38.03 \\
$u_{0}$ & 355.65 & 374.24 & 369.63 & 51.78 \\
$v_{0}$ & 233.76 & 255.71 & 270.06 & 34.32
\end{tabular}

In order to obtain the ground truth values, we applied the calibration from pattern method in [1819], using all the "chessboard" patterns in the five images. The ground truth angle between the sensor axes was $\theta=89.42$ degrees, and the ground truth aspect ratio was $k_{v} / v_{u}=1.01$. Therefore, the assumption of unit aspect ratio can be considered as valid for the camera. The ground truth parameters of the focal lengths $\alpha_{u}, \alpha_{v}$ and the camera centre $\left(u_{0}, v_{0}\right)$ were:

$$
\alpha_{u}=856.33, \alpha_{v}=865.86, u_{0}=385.24, v_{0}=273.22 \text {. }
$$

We observe that, even if the square pixel assumption was not completely correct for the actual camera, the obtained calibration was close to its ground truth.

\section{Conclusion}

We have shown that the parallel screw axis camera motion can be used in order to obtain image sequences easy to self-calibrate, being this motion a possible substitute or complement to planar camera motions. Our method complexity is not higher than cubic: after finding the unique real root of certain univariate cubics, two linear steps are performed to achieve the camera self-calibration.

The proposed stratified self-calibration method has been demonstrated to give good results on both synthetic and real data. Experiments on synthetic images have shown that better results are obtained when the camera is oriented far from being parallel to the screw direction. The addition of a roll angle to the camera also improves the estimation of the affine and Euclidean reconstructions.

The study of the resilience of the method to deviations from the assumption of parallel rotation axes could be a further topic of research. An algebraic characterisation of those camera motions leading to imaged horopters intersecting in four points has been omitted in this paper, due to lack of space; its geometric interpretation is missing and would also be desirable.

\section{References}

1. Armstrong, M., Zisserman, A., Hartley, R.I.: Self-calibration from image triplets. In: Buxton, B.F., Cipolla, R. (eds.) ECCV 1996. LNCS, vol. 1065, Springer, Heidelberg (1996) 
2. Demirdjian, D., Csurka, G., Horaud, R.: Autocalibration in the presence of critical motions. In: Proc. BMVC (1998)

3. Demirdjian, D., Zisserman, A., Horaud, R.: Stereo autocalibration from one plane. In: Vernon, D. (ed.) ECCV 2000. LNCS, vol. 1843, pp. 625-639. Springer, Heidelberg (2000)

4. Engels, C., Stewénius, H., Nistér, D.: Bundle adjustment rules. In: PCV (2006)

5. Espuny, F.: A new linear method for camera self-calibration with planar motion. J. Math. Imag. Vis. 27(1), 81-88 (2007)

6. Faugeras, O., Luong, Q.T., Papadopoulou, T.: The Geometry of Multiple Images: The Laws That Govern The Formation of Images of A Scene and Some of Their Applications. MIT Press, Cambridge (2001)

7. Faugeras, O., Quan, L., Sturm, P.: Self-calibration of a 1D projective camera and its application to the self-calibration of a $2 \mathrm{D}$ projective camera. In: Burkhardt, H.J., Neumann, B. (eds.) ECCV 1998. LNCS, vol. 1406, p. 36. Springer, Heidelberg (1998)

8. Habed, A., Boufama, B.: Camera self-calibration: A new approach for solving the modulus constraint. In: Proc. ICPR (2004)

9. Hartley, R.I.: Self-calibration from multiple views with a rotating camera. In: Eklundh, J.-O. (ed.) ECCV 1994. LNCS, vol. 801, Springer, Heidelberg (1994)

10. Hartley, R.I., de Agapito, L., Reid, I.D., Hayman, E.: Camera calibration and the search for infinity. In: Proc. ICCV (1999)

11. Hartley, R.I., Zisserman, A.: Multiple View Geometry in Computer Vision, 2nd edn. Cambridge University Press, Cambridge (2004)

12. Knight, J., Zisserman, A., Reid, I.: Linear auto-calibration for ground plane motion. In: Proc. CVPR (2003)

13. Manning, R.A., Dyer, C.R.: Stratified self calibration from screw-transform manifolds. In: Heyden, A., Sparr, G., Nielsen, M., Johansen, P. (eds.) ECCV 2002. LNCS, vol. 2353, pp. 131-145. Springer, Heidelberg (2002)

14. Pollefeys, M., Van Gool, L., Oosterlinck, A.: The modulus constraint: A new constraint for self-calibration. In: Proc. ICPR (1996)

15. Ronda, J.I., Valdés, A., Jaureguizar, F.: Camera autocalibration and horopter curves. Int. J. Comput. Vis. 57(3), 219-232 (2004)

16. Schaffalitzky, F.: Direct solution of modulus constraints. In: Proc. ICVGIP (2000)

17. Sturm, P.: Critical motion sequences for monocular self-calibration and uncalibrated Euclidean reconstruction. In: Proc. CVPR (1997)

18. Sturm, P., Maybank, S.J.: On plane-based camera calibration: A general algorithm, singularities, applications. In: Proc. CVPR (1999)

19. Zhang, Z.: Flexible camera calibration by viewing a plane from unknown orientations. In: Proc. ICCV (1999)

20. Zisserman, A., Liebowitz, D., Armstrong, M.: Resolving ambiguities in autocalibration. Phil. Trans. Roy. Soc. Lond. Math. Phys. Sci. 356(1740), 1193-1211 (1998) 